\title{
Quantitative synchrotron micro-XRF study of CoTSPc and CuTSPc thin-films deposited on gold by cyclic voltammetry
}

\author{
Karl Peeters, ${ }^{a}$ Karolien De Wael, $\dagger^{a}$ Annemie Adriaens, ${ }^{* a}$ Gerald Falkenberg ${ }^{b}$ and \\ Laszlo Vincze ${ }^{a}$
}

Received 6th November 2006, Accepted 26th February 2007

First published as an Advance Article on the web 12th March 2007

DOI: $10.1039 / \mathrm{b616167g}$

Synchrotron radiation X-ray fluorescence (SR-XRF) spectroscopy has been applied for the microscopic characterization of cobalt(II) tetrasulfonated phthalocyanine (CoTSPc) and copper(II) $3,4^{\prime}, 4^{\prime \prime}, 4^{\prime \prime \prime}$-tetrasulfonated phthalocyanine (CuTSPc) thin films electrochemically deposited on gold electrodes. The deposited thin-film properties have been studied as a function of phthalocyanine concentration in solution during the modification process. The $\mathrm{Co}$ and $\mathrm{Cu}$ surface concentrations on the modified gold electrodes have been determined on the 20 and $600 \mu \mathrm{m}$ levels. The SR-XRF quantification procedure and micro-heterogeneity determination of the CoTSPc and CuTSPc thin-films on the gold electrodes have been described in detail. The detailed comparison of scanning SR-XRF results with electrochemical data makes it possible to gain further insight in the mechanism of thin layer growth during the modification procedure.

\section{Introduction}

Transition metal metallophthalocyanines are commonly used in high-tech applications, such as in photosensitizers, optical data storage and chemical gas sensors, ${ }^{1-5}$ and have lately attracted interest in the field of electrochemistry where they are being tested and used as electrocatalysts. ${ }^{6-10}$ Soluble sulfonated phthalocyanines have shown themselves to be able to form stable layers on different types of supporting material, including electrodes. The immobilization of these compounds onto an electrode can lead to so-called modified electrodes with improved kinetic properties, allowing a rate increase in an electrochemical reaction in order to provide a higher selectivity, sensitivity and efficiency. The major characteristics of the phthalocyanines are their high thermal and chemical stability, coupled with their extensive redox chemistry. Moreover, the phthalocyanine ring is a flat structure, which makes the aggregation in solution and on surfaces possible.

Various deposition techniques have been used to create modified electrodes, including drop drying, spin coating, spontaneous adsorption (self-assembling) and electrodeposition. ${ }^{11-14}$ In previous studies synchrotron radiation X-ray micro-fluorescence (SR-XRF) was used to study the morphology and heterogeneity of cobalt(II) tetrasulfonated phthalocyanine (CoTSPc) and copper(II) 3,4',4", $4^{\prime \prime \prime}$-tetrasulfonated phthalocyanine (CuTSPc) on gold electrodes using the different modification techniques. ${ }^{15}$ Results demonstrated that the

\footnotetext{
${ }^{a}$ Ghent University, Department of Analytical Chemistry, Krijgslaan 281 S12, B-9000 Ghent, Belgium. E-mail: annemie.adriaens@ugent.be; Fax: +3292644960;TTel: +329264 4826

${ }^{b}$ Hamburger Synchrotronstrahlungslabor at Deutsches ElektronenSynchrotron, DESY, D-22607 Hamburg, Germany

$\dagger$ Postdoctoral Fellow of the Research Foundation-Flanders (FWO), Belgium.
}

electrodeposition technique leads to a more efficient adsorption, resulting in a uniformly adsorbed phthalocyanine layer.

This paper focuses on the quantitative aspects of SR-XRF (micro-)characterization of CoTSPc and CuTSPc thin-films which are electrochemically deposited on gold electrodes. The derived $\mathrm{Co}$ and $\mathrm{Cu}$ surface-concentration results enable the quantitative assessment of CoTSPc and CuTSPc thin film build-up on both macro- and microscopic scales after electrodeposition as a function of the applied phthalocyanine concentration. The SR-XRF based surface concentration values and their microscopic variation across the electrode surface have been derived using the data collection and reduction strategy described by Kempenaers et al., ${ }^{16-18}$ coupled with a calibration scheme based on the fundamental parameter method.

Microbeam SR-XRF allowed the quantitative determination of the heterogeneity of CoTSPc or CuTSPc surface concentrations on the $20 \mu \mathrm{m}$ and $600 \mu \mathrm{m}$ level. The obtained mean concentration values could be compared directly with electrochemical calculations, yielding satisfactory agreement between the two determination methods for the deposited surface concentration values and new insights into CoTSPc and CuTSPc thin film build-up.

\section{Experimental}

\section{Electrodeposition procedure}

The electrodeposition was carried out in a three electrode cell with a saturated calomel reference electrode (SCE) with two compartments, obtained from Radiometer (Lyon, France), and a carbon counter electrode. The working electrode was a gold electrode (diameter $1.6 \mathrm{~mm}$ ) from BAS (Warwickshire, UK), which was pre-treated by mechanical and electrochemical polishing according to the following procedure. Before its first use the electrode surface was briefly scoured by a $\mathrm{SiC}$ 
emery paper, 1200 grit, to obtain a fresh surface. To smoothen the resulting relatively rough surface it was further subjected to sequential polishing by a polishing cloth covered with alumina powder (Buehler, IL, USA) of $1,0.3$ and $0.05 \mu \mathrm{m}$ particle size for, respectively, 5, 10 and $20 \mathrm{~min}$. To remove any adherent $\mathrm{Al}_{2} \mathrm{O}_{3}$ particles the electrode surface was rinsed thoroughly with doubly de-ionised water and cleaned in an ultrasonic bath (Branson 3210, Ghent, Belgium) containing de-ionised water for $2 \mathrm{~min}$. Finally, the electrode was pretreated using an electrochemical potential cycling technique in a $0.1 \mathrm{~mol} \mathrm{~L}^{-1} \mathrm{Na}_{2} \mathrm{HPO}_{4}-\mathrm{NaOH}$ buffer solution, $\mathrm{pH}=12$, between -1.2 and $0.6 \mathrm{~V}$ versus $\mathrm{SCE}$ until 5 subsequent voltammetric scans were identical.

In the next step, the electrochemical potential cycling technique was used to deposit a film of phthalocyanines onto a gold electrode. The modification was carried out by recording successive cyclic voltammetric scans in a potential window from -1.2 to $0.6 \mathrm{~V}$ versus $\mathrm{SCE}\left(50 \mathrm{mV} \mathrm{s}^{-1}\right)$ during a defined number of voltammetric scans in a $\mathrm{pH} 12$ buffer solution containing either CoTSPc or CuTSPc. The modified gold electrodes were kept in a phthalocyanine free $\mathrm{pH} 12$ buffer solution and 20 voltammetric scans were taken between -1.2 and $0.6 \mathrm{~V}$ versus SCE with a scan rate of $50 \mathrm{mV} \mathrm{s}^{-1}$. The surface concentration of phthalocyanine on the gold electrode is derived from the $\mathrm{Co}(\mathrm{III}) \mathrm{TSPc}_{\mathrm{ads}} / \mathrm{Co}$ (II)TSPc $\mathrm{Tds}_{\mathrm{ads}}$ reduction peak $(-0.38 \mathrm{~V}$ versus SCE) for the electrode modified with CoTSPc and from the $\mathrm{Cu}(\mathrm{I}) \mathrm{TSP}_{\mathrm{ads}} / \mathrm{Cu}(\mathrm{II}) \mathrm{TSP}_{\mathrm{ads}}$ oxidation peak $(0.05 \mathrm{~V}$ versus SCE) for the CuTSPc modified electrode. ${ }^{14,15,19,20}$

A PGSTAT20 potentiostat from ECO Chemie controlled by GPES 4.9 software package running on a Pentium II computer (Eknadata) was used to record the voltammetric curves. Before use, measurement of the $\mathrm{pH}$ of the solution was made using an Orion Benchtop pH-meter, Model 420A.

During this study the concentrations of CoTSPc or CuTSPc were varied from 1 to $16 \mathrm{mmol} \mathrm{L}^{-1}$ in a $\mathrm{pH} 12$ buffer solution. The CoTSPc sodium salts, having four sulfonated groups randomly placed over the phthalocyanine structure, were purchased from the Department of Chemistry at Rhodes University of Grahamstown (Eastern Cape, South Africa). The CuTSPc sodium salts were purchased from SigmaAldrich (St. Louis, USA). In the case of the latter, the four sulfonated groups are on fixed positions, more specifically on the $3,4^{\prime}, 4^{\prime \prime}, 4^{\prime \prime \prime}$ spot of a phthalocyanine ring (CuTSPc). The buffer solution of $\mathrm{pH} 12\left(\mathrm{Na}_{2} \mathrm{HPO}_{4}-\mathrm{NaOH}\right)$ was purchased from VWR-International (Leuven, Belgium). Before each experiment, pure nitrogen was bubbled through the cell solution for $20 \mathrm{~min}$.

\section{Scanning SR-XRF measurements}

The scanning micro-SR-XRF experiments were performed at Beam Line $\mathrm{L}$ of the DORIS-III storage ring, HASYLAB (Hamburg, Germany). ${ }^{21}$ This beamline is dedicated to micro-XRF experiments using either white or monochromatic bending magnet excitation and mono- or polycapillary focusing, with routinely available beam sizes of $10-30 \mu \mathrm{m}$. The primary X-ray beam is generated by a $1.2 \mathrm{~T}$ bending magnet source which, given the machine energy of $4.465 \mathrm{GeV}$, pro- vides a polychromatic spectral distribution with a critical energy of $16.6 \mathrm{keV}$. After initial low-energy filtering and collimation, the beam was monochromatized by a $\mathrm{Ni} / \mathrm{C}$ multi-layer monochromator $\left(\Delta E / E \approx 10^{-2}\right)$ and further collimated by a motorized cross-slit system. After monochromatization the beam was focused by a monolithic polycapillary half-lens (X-ray Optical Systems Inc., USA). This optic is designed specifically for parallel primary beams and hence is suitable for the focusing of low-divergence synchrotron beams. A microbeam size of about $20 \mu \mathrm{m}$ (FWHM) was obtained at the excitation energy range of $8.0 \pm 0.1 \mathrm{keV}$ or $9.3 \pm 0.1 \mathrm{keV}$ (beyond the XANES region with the strongest oscillations), which was used to excite, respectively, the $\mathrm{Co} K \alpha, \beta$ or the $\mathrm{Cu}$ $\mathrm{K} \alpha, \beta$ fluorescent-lines without exciting $\mathrm{Au} \mathrm{L}$ lines from the (spectroscopically infinitely thick) gold substrate. The nominal working distance of the polycapillary optic was $5 \mathrm{~mm}$. An energy dispersive VORTEX Si-drift detector was used for $\mathrm{X}$-ray detection, having an active area of $50 \mathrm{~mm}^{2}$.

The experimental difficulty of the CoTSPc or CuTSPc thinfilm uniformity studies included the determination of low amounts of $\mathrm{Co}$ or $\mathrm{Cu}$ (ca. 0.1-0.5 pg, assuming a $20 \mu \mathrm{m}$ incident beam) in the presence of partially overlapping Compton/Rayleigh and Au X-ray resonant Raman peaks. An example of the SR-XRF sum spectra obtained from a gold electrode with deposited CoTSPc (a) or CuTSPc (b) thin-films is shown in Fig. 1. During the CuTSPc uniformity studies $31 \times$ 31 scanning XRF maps were recorded with a step-size of $20 \mu \mathrm{m}$, using a data collection time of $10 \mathrm{~s}$ per data point. The intensities of the incoming beam were monitored using an ionization chamber in front of the polycapillary.

The SR-XRF measurements were performed on the gold electrodes modified with CoTSPc in a concentration range from 1 to $16 \mathrm{mmol} \mathrm{L}^{-1}$. In the concentration range of 1-10 mmol L ${ }^{-1}$, every modification was performed until the number of voltammetric scans needed to reach the stabilization point, also called the breaking point, was reached ${ }^{14}$ (see below) in order to receive the same state of the immobilized layer. In the concentration range of $12-16 \mathrm{mmol} \mathrm{L}^{-1}$ the electrodeposition was performed until beyond the breaking point. ${ }^{14}$ The reason for this is that the number of voltammetric scans needed to reach the breaking point is lower than five for these CoTSPc concentrations, which has a negative influence on the reproducibility of the modification. For the electrodeposition with

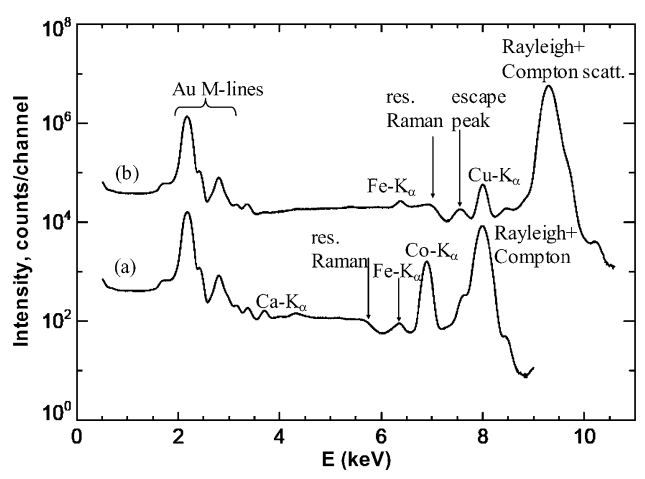

Fig. 1 SR-XRF spectrum of a gold electrode electrochemically modified with CoTSPc (a) or CuTSPc (b). 
CuTSPc, 60 subsequent voltammetric scans were taken during the modification in a concentration range of $1-16 \mathrm{mmol} \mathrm{L}^{-1}$.

\section{Data reduction}

The non-linear least square fitting software $\mathrm{AXIL}^{22}$ was used to determine the net-peak intensities of the $\mathrm{Co} \mathrm{K} \alpha$ or $\mathrm{Cu} \mathrm{K} \alpha$ in the measured XRF spectra. The average deposited surface concentrations were calculated from the sum spectrum by the fundamental parameter (FP) method, ${ }^{23}$ making use of pure thin $\mathrm{Co}$ (thickness $10 \mu \mathrm{m}$ ) and $\mathrm{Cu}$ (thickness $1 \mu \mathrm{m}$ ) foils as standards for the calibration (Goodfellow Inc.). The data reduction described below aims at the determination of the average surface concentration values and their relative variance $\delta_{\text {sum,r }}^{2}$ based on the sum spectra for the individual Co or $\mathrm{Cu}$ concentrations on the gold electrode. These values on the one hand are influenced by the relative variance of the experimental $\mathrm{Co}$ or $\mathrm{Cu}$ intensities originating from the deposited thin-films on the gold electrode. On the other hand, the uncertainty of the determined thin-film concentration also depends on the relative variance of the measured $\mathrm{Co}$ and $\mathrm{Cu}$ intensities from the applied standards used for calibration/ normalization.

The concentrations of $\mathrm{Co}$ and $\mathrm{Cu}$ calculated by the FP method have been plotted as a function of the $\mathrm{Au} M$ line normalized $\mathrm{Co} \mathrm{K} \alpha$ or $\mathrm{Cu} \mathrm{K} \alpha$ intensities $\left(I_{\mathrm{Co}-\mathrm{K} \alpha} / I_{\mathrm{Au}-\mathrm{M}}\right.$ or $I_{\mathrm{Cu}-\mathrm{K} \alpha} / I_{\mathrm{Au}-\mathrm{M}}$ ) derived from the sum spectra (Fig. 2). In this way, a calibration curve for $\mathrm{Co}$ and $\mathrm{Cu}$ could be obtained.

During one set of experiments (see below) this calibration curve was used to quantify the data, which simplified the quantification procedure considerably by using the gold Mlines as internal reference, without need of using external standards. Instead of the direct application of the fundamental parameter method, the calibration curves shown in Fig. 2 were used to derive the surface concentrations of the Co or the $\mathrm{Cu}$ on the gold electrodes from the sum spectra. This resulted in an increase of $\delta_{\text {sum,r }}^{2}$ for Co and a decrease of $\delta_{\text {sum,r }}^{2}$ for $\mathrm{Cu}$. Since the energy of the incoming beam was shifted to more positive values ( $c a .260 \mathrm{eV}$ for $\mathrm{Co}$ and $390 \mathrm{eV}$ for $\mathrm{Cu}$ ) for this set of experiments compared with those from which the calibration curves were derived, a sensitivity correction term

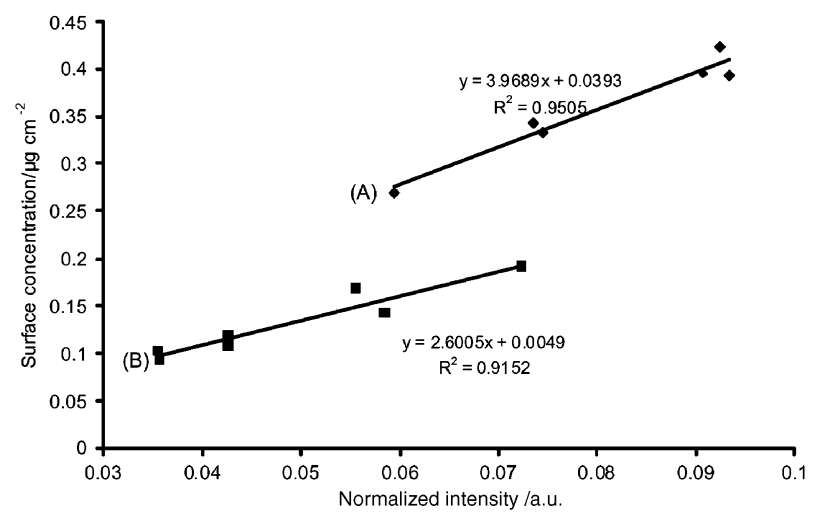

Fig. 2 Calibration curve of cobalt (A) and copper (B) concentrations on the gold electrode surface as a function of the total $\mathrm{Co}$ or $\mathrm{Cu}$ intensity derived from the sum spectrum after normalization with the gold $\mathrm{M}$ lines. was applied. The sensitivity correction term for these shifts has been determined by using the corresponding mass attenuation coefficients derived from the McMaster tabulation. ${ }^{24}$

The Co or $\mathrm{Cu}$ surface concentrations on the pixel level were also derived from the 961 individual spectra (representing a $31 \times 31$ SR-XRF map), each of which corresponds to concentration values from microscopic regions on the $20 \mu \mathrm{m}$ scale. Within the $31 \times 31$ elemental maps, the individual pixel intensities represent the $\mathrm{Co} \mathrm{K} \alpha$ or $\mathrm{Cu} \mathrm{K} \alpha$ line-intensities normalized by the $\mathrm{Au} \mathrm{M}$ lines (after integration between $1.862 \mathrm{keV}$ and $2.362 \mathrm{keV}$ ). From these normalized SR-XRF maps, corresponding histograms of the detected $\mathrm{Co}$ and $\mathrm{Cu}$ signals were derived. By fitting Gaussian curves on these histograms the mean value and the full-width-at-half-maximum (FWHM) of the measured intensity distributions have been obtained. According to Kempenaers et al., ${ }^{16-18}$ the relative total variance $\delta_{\text {tot,r }}^{2}$ calculated on these 961 pixels can be attributed to the relative statistical variance $\delta_{\text {stat, r }}^{2}$, the relative instrumental variance $\delta_{\text {instr, } r}^{2}$ and the relative heterogeneity $\delta_{\text {het, } \mathrm{r}}^{2}$ terms, according to the equation $\delta_{\text {tot, } \mathrm{r}}^{2}=\delta_{\text {stat }, \mathrm{r}}^{2}+$ $\delta_{\text {instr,r }}^{2}+\delta_{\text {het, } \mathrm{r}}^{2}$.

Using the mean values of these histograms and the calibration curve from the sum spectra, the mean $\mathrm{Co}$ and $\mathrm{Cu}$ concentrations were obtained on the individual pixel level. By use of the FWHM of the derived histograms, the total variance $\delta_{\text {tot, r }}^{2}$ can be calculated, which can be divided into contributions of statistical, instrumental and heterogeneity variance terms (respectively $\delta_{\text {stat,r },}^{2}, \delta_{\text {instr, r }}^{2}$ and $\delta_{\text {het,r }}^{2}$ ) of the sample, according to Kempenaers et al. ${ }^{17}$ The instrumental term $\delta_{\text {instr,r } r}^{2}$ is considered to be negligible here since it is smaller than $1 \%$ for the given experimental setup. ${ }^{18}$ The statistical term $\delta_{\text {stat,r }}^{2}$ can be derived from the effective counts according to the $\mathrm{Co}$ or $\mathrm{Cu}$ signal of the sum spectrum, assuming Poisson statistics. With these assumptions, the heterogeneity term of interest $\delta_{\text {het,r }}^{2}$ can be derived as the difference of the measured total variance term $\delta_{\text {tot,r }}^{2}$ and that of the statistical variance term $\delta_{\text {stat,r. }}^{2}$.

\section{Results}

\section{SR-XRF and electrochemical determination of CoTSPc or CuTSPc surface concentrations deposited on a gold electrode}

In previous work it has been shown that the charge related to the adsorption process of CoTSPc, which is calculated by using the surface area of the $\mathrm{Co}$ (III) $\mathrm{TSPc}_{\mathrm{ads}} / \mathrm{Co}(\mathrm{II}) \mathrm{TSPc}_{\mathrm{ads}}$ reduction peak, first increases with the growing number of voltammetric scans and then becomes constant. ${ }^{14}$ The number of voltammetric scans needed to reach the breaking point is also concentration dependent. ${ }^{14}$ It has been postulated that prior to this breaking point, the adsorption is kinetically controlled, resulting in a chaotic multilayer, and once a saturation of the deposited CoTSPc has been obtained, a reorganization of the chaotic multilayer takes place towards a more stable and ordered structure. ${ }^{14,15,19}$ In comparison with the Co data, the charge related to the adsorption process of CuTSPc shows a different behaviour. The charge keeps increasing with growing voltammetric scan number. This means that from voltammetric scan 1, the CuTSPc molecules are 
Table 1 Overview of the electrochemical data including, from left to right, the number of the measurement, the number of subsequent voltammetric scans in a pH 12 buffer solution containing either CoTSPc or CuTSPc (the asterix $\left(^{*}\right)$ symbol indicates that this number of voltammetric scans is the breaking point for the given concentration), the concentration CoTSPc or CuTSPc in the solution during the modification, the charge due to the $\mathrm{Co}$ (III) $/ \mathrm{Co}$ (II) or $\mathrm{Cu}(\mathrm{II}) / \mathrm{Cu}(\mathrm{I})$ adsorption peak and the $\mathrm{Co}$ or $\mathrm{Cu}$ surface concentration

\begin{tabular}{lllll}
\hline & $\begin{array}{l}\text { Number of } \\
\text { voltammetric } \\
\text { scans }\end{array}$ & $\begin{array}{l}\text { Phthalocyanine } \\
\text { concentration/ } \\
\text { mmol L }^{-1}\end{array}$ & $\begin{array}{l}\text { Charge/ } \\
\mu \mathrm{C}\end{array}$ & $\begin{array}{l}\text { Surface } \\
\text { concentration/ } \\
\mu \mathrm{g} \mathrm{cm}^{-2}\end{array}$ \\
\hline CoTSPc & & & & \\
1 & $27^{*}$ & 1.0 & 4.7 & 0.143 \\
2 & $25^{*}$ & 2.0 & 4.5 & 0.137 \\
3 & $21^{*}$ & 4.0 & 4.7 & 0.143 \\
4 & $17^{*}$ & 6.0 & 4.9 & 0.148 \\
5 & $13^{*}$ & 8.0 & 5.4 & 0.163 \\
6 & $9 *$ & 10.0 & 4.5 & 0.136 \\
7 & 9 & 12.0 & 3.2 & 0.097 \\
8 & 9 & 14.0 & 6.3 & 0.191 \\
9 & 9 & 16.0 & 5.0 & 0.151 \\
& & & & \\
$\mathrm{CuTSPc}$ & & 1.0 & 2.8 & 0.094 \\
10 & 60 & 2.0 & 2.1 & 0.099 \\
11 & 60 & 4.0 & 3.9 & 0.144 \\
12 & 60 & 6.0 & 3.8 & 0.179 \\
13 & 60 & 8.0 & 4.3 & 0.141 \\
14 & 60 & 10.0 & 5.5 & 0.123 \\
15 & 60 & 12.0 & 4.4 & 0.128 \\
16 & 60 & 14.0 & 3.0 & 0.069 \\
17 & 60 & 16.0 & 2.9 & 0.091 \\
18 & 60 & & & \\
\hline
\end{tabular}

stacked in their definitive position and that there is no further reorganization of the immobilized layer. ${ }^{15,20}$

Table 1 lists the parameters of the modification procedure used in this study together with the electrochemical data obtained and the derived surface concentrations for the modification with CoTSPc as well as with CuTSPc. The voltammetric scan numbers labelled with an asterisk (*) symbol in Table 1 indicates that the modification occurred up to the breaking point for this CoTSPc concentration in solution. From Table 1 it can be seen that the calculated Co surface concentration is independent of the CoTSPc concentration during the modification process at the breaking point (measurements 1-6) and after the breaking point (measurements 7-9) in the concentration range studied. It can also be seen that the deposited $\mathrm{Cu}$ surface concentration is more or less independent of the initial CuTSPc concentration in solution in the concentration range of $1-16 \mathrm{mmol} \mathrm{L}^{-1}$ during the modification procedure after 60 subsequent voltammetric scans.

The SR-XRF based surface concentration values of CoTSPc or CuTSPc were derived from the sum spectra (yielding the average concentration) as well as from the 961 individual spectra (yielding the concentration at the microscopic level). From the sum spectra a calibration curve has been plotted (Fig. 2) showing that the $I_{\mathrm{Cu}-\mathrm{K} \alpha} / I_{\mathrm{Au}-\mathrm{M}}$ ratio, shown in the $X$-axis of the calibration curve, is higher than the $I_{\mathrm{Co}-\mathrm{K} \alpha} / I_{\mathrm{Au}-\mathrm{M}}$ ratio because of the higher excitation energy used during the $\mathrm{Cu}$ detection. The higher excitation energy reduces considerably the gold $\mathrm{M}$-line intensity (used as the internal reference) during $\mathrm{Cu}$ detection, which results in a higher normalised $\mathrm{Cu} / \mathrm{Au}-\mathrm{M}$ signal for the same $\mathrm{Cu}$ or $\mathrm{Co}$ surface concentration. The standard deviations of the measured $\mathrm{Co}$ and $\mathrm{Cu}$ concentrations in the calibration curve are, respectively, $0.0139 \mu \mathrm{g} \mathrm{cm}^{-2}$ and $0.0118 \mu \mathrm{g} \mathrm{cm}^{-2}$.

The normalized elemental maps of the deposited Co thin films and the corresponding histograms of the normalized Co$\mathrm{K} \alpha$ intensities for different CoTSPc concentrations in the solution are shown in Fig. 3. The equivalent XRF maps and histograms for $\mathrm{Cu}$ are shown in Fig. 4, corresponding to a modification of 60 voltammetric scans as a function of CuTSPc concentration. The histograms shown in these figures have been used to derive the surface concentration values and their variances listed in Table 2, which provides an overview of the results obtained from the SR-XRF experiments. The table shows the total $\mathrm{Co}$ or $\mathrm{Cu}$ intensities, the normalized $\mathrm{Co}$ or $\mathrm{Cu}$ intensities and the surface concentration values $\delta_{\text {sum,r }}$ derived from the sum spectrum. Also indicated are the (microscopic) mean surface concentrations obtained from the mean of the histograms, as well as the quantities $\delta_{\text {tot,r }}, \delta_{\text {stat,r }}$, and $\delta_{\text {het }}$, defined earlier. The numbering of the experiments is the same as the numbering used in Table 1. Comparison of the average $\mathrm{Co} / \mathrm{Cu}$ surface concentrations and the $\mathrm{Co} / \mathrm{Cu}$ concentration on the microscopic level shows that the $\mathrm{Co} / \mathrm{Cu}$ concentration on the microscopic level is slightly smaller. This can be explained by the detected heterogeneities on the deposited layer having high concentrations, which can be seen as small bright areas on the recorded elemental maps. These areas correspond to the high-intensity tails on the right-hand side of the calculated histograms, representing in some cases considerable deviations from the ideal Gaussian distribution. It is also seen in Table 2 that $\delta_{\text {sum,r }}$ is about 40 times higher for $\mathrm{Cu}$ than for Co. This can be explained by the considerably higher waviness of the thinner $(1 \mu \mathrm{m})$ copper foil used as standard compared with the Co reference foil $(10 \mu \mathrm{m})$, resulting in higher uncertainties of the illuminated excitation volume (and detected reference intensity) for $\mathrm{Cu}$.

Fig. 5 shows the elemental maps of $\mathrm{Co}$ or $\mathrm{Cu}$ deposited using different CoTSPc ( 9 voltammetric scans) or CuTSPc (60 voltammetric scans) concentrations in solution and the corresponding histograms of the normalized $\mathrm{Co}-\mathrm{K} \alpha$ or $\mathrm{Cu}-\mathrm{K} \alpha$ intensities derived from another set of experiments. For the determination of the surface concentrations from these SR-XRF measurements, the calibration curve shown in Fig. 2 has been used. In this case the surface concentrations could be derived directly from the $I_{\mathrm{Co}-\mathrm{K} \alpha} / I_{\mathrm{Au}-\mathrm{M}}$ or $I_{\mathrm{Cu}-\mathrm{K} \alpha} / I_{\mathrm{Au}-\mathrm{M}}$ ratios without the use of an external standard. It can be seen in Table 2 (measurements 7, 8, 9, 17 and 18) that the use of internal reference results in increased $\delta_{\text {sum,r }}$ values for Co, while the $\delta_{\text {sum,r }}$ reduces for $\mathrm{Cu}$ when compared with the FP quantification using an external reference. This can be explained by the relatively high $\delta_{\text {sum,r }}$ values for $\mathrm{Cu}$ in the case of the external standard based quantification as a result of waviness of the thin $\mathrm{Cu}$ reference foil $(1 \mu \mathrm{m})$. In the case of the quantification of $\mathrm{Co}$, the standard deviation on the calibration curve dominates when compared with the negligible standard deviation measured from the external standard foil $(10 \mu \mathrm{m})$. In this case switching from external reference to the calibration curve based quantification increases $\delta_{\text {sum,r. }}$.

In the rest of this paper, the surface concentrations derived from the electrochemical data (Table 1) are compared with the 

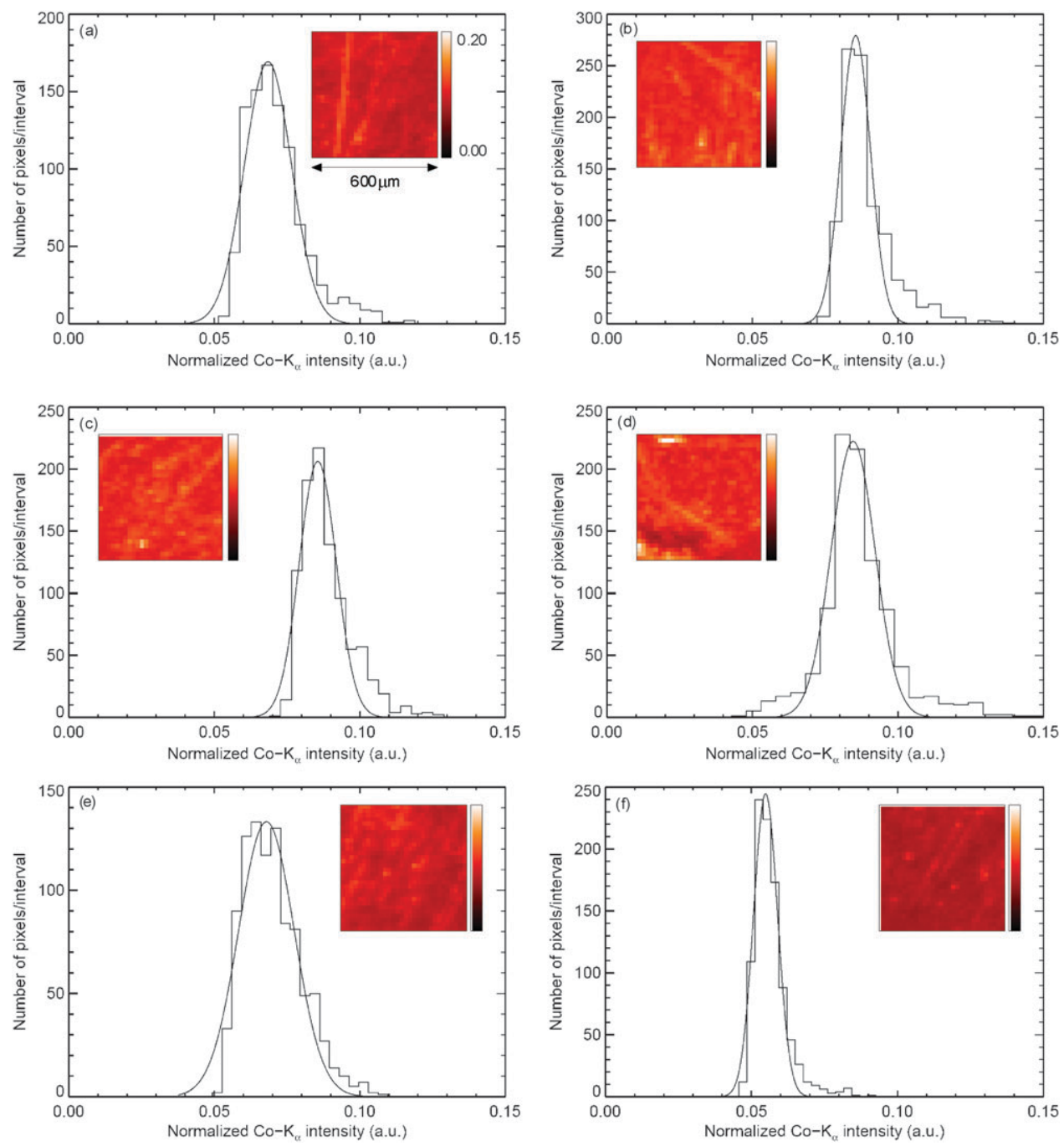

Fig. 3 Elemental maps of deposited Co and the corresponding histogram of the detected Co K $\alpha$ line obtained from a modified gold electrode at the breaking point as a function of the concentration of CoTSPc in solution: (a) 1, (b) 2, (c) 4, (d) 6 , (e) 8 and (f) $10 \mathrm{mmol} \mathrm{L}^{-1}$. The XRF maps consist of a $31 \times 31$ raster using $20 \mu \mathrm{m}$ step size in both $X$ and $Y$ directions and the scale bar is the same for all the maps, indicating a normalized Co-K $\alpha$ intensity between 0 and 0.2 .

surface concentrations derived from the SR-XRF data (Table 2). This comparison gives detailed information about the layer build-up of the CoTSPc or CuTSPc thin layer, which is discussed in the next section.

\section{Discussion}

\section{Comparison of the Co surface concentrations on gold electrodes} derived from SR-XRF and electrochemical data

Fig. 6 shows the Co surface concentrations calculated using the electrochemical data, the SR-XRF sum spectra and using the mean of the histogram as a function of the CoTSPc concentration $\left(1-10 \mathrm{mmol} \mathrm{L}^{-1}\right)$. The number of voltammetric scans during the modification is indicated each time between parentheses. It is observed that the surface concentrations derived from the electrochemical data, as well as from the SR-XRF data, are reasonably constant across the concentration range studied. Moreover, it is seen that the Co surface concentration derived from the electrochemical data is significantly lower than those obtained from the SR-XRF data. This constant difference between the electrochemical and SR-XRF surface concentrations means that there is a constant ratio between the electro-active (electrochemical data) and electroinactive species on the surface at the breaking point. Note that the SR-XRF data show both electrochemically active as well as the electrochemically inactive species.

Fig. 7 shows the surface concentrations of cobalt on a gold electrode derived from the electrochemical and SR-XRF data after a modification using nine voltammetric scans with the CoTSPc concentration varying between 10 and $16 \mathrm{mmol} \mathrm{L}^{-1}$ in the solution. Here, the micro-heterogeneity term $\left(\delta_{\text {het }}\right)$ is represented by the error bars on the concentrations derived from the mean of the histograms. For the CoTSPc concentrations of 12,14 and $16 \mathrm{mmol} \mathrm{L}^{-1}$, nine voltammetric scans are beyond the breaking point; for the CoTSPc concentration of $10 \mathrm{mmol} \mathrm{L}^{-1}$, on the other hand, this is just at the breaking point. Based on earlier studies, ${ }^{14}$ it is expected that the cobalt 

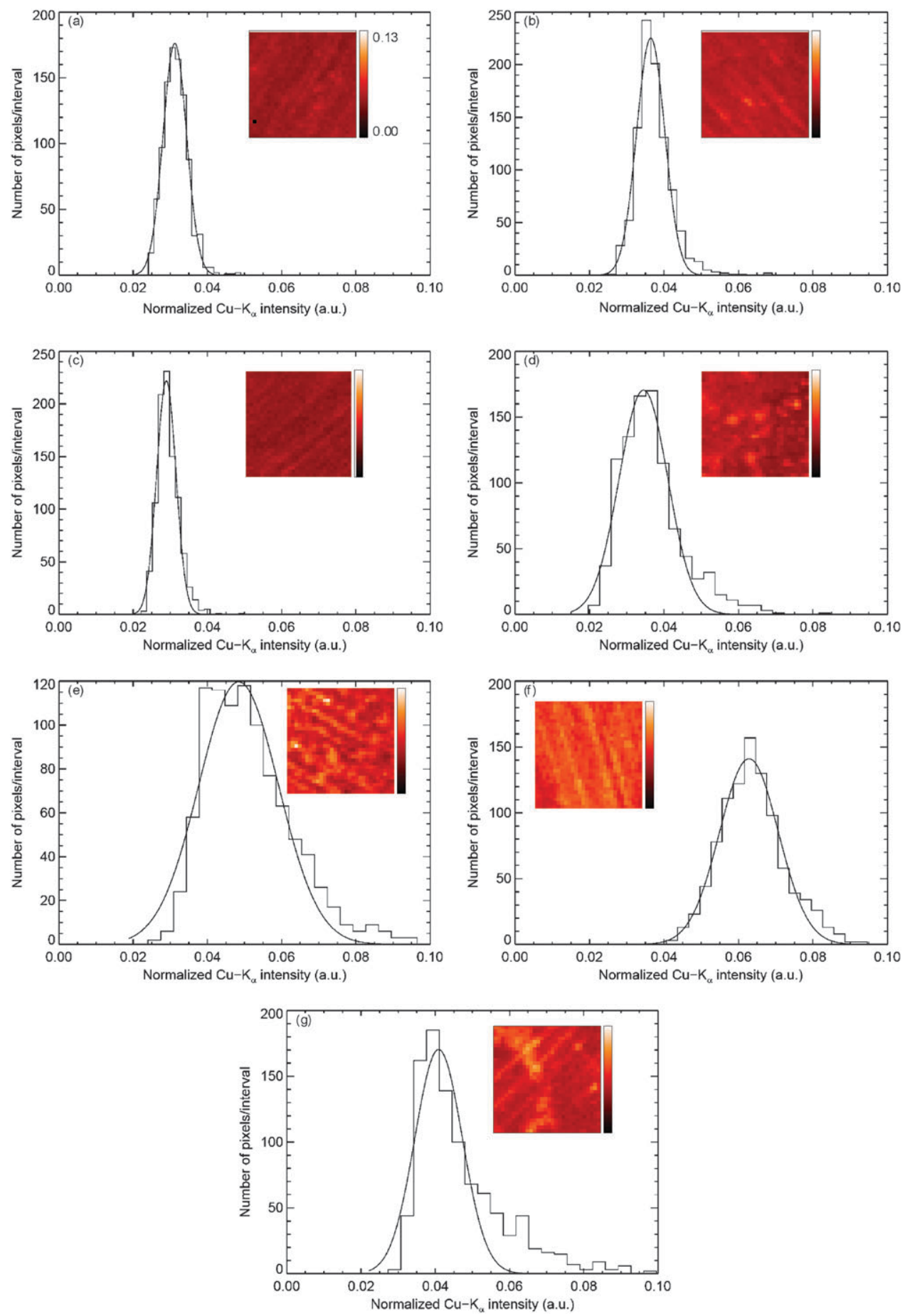

Fig. 4 Elemental maps of deposited $\mathrm{Cu}$ and the corresponding histogram of the detected $\mathrm{Cu} \mathrm{K} \alpha$ line obtained from a modified gold electrode at the breaking point as a function of the concentration of CoTSPc in solution: (a) 1, (b) 2, (c) 4, (d) 6, (e) 8, (f) 10 and (g) 12 mmol L ${ }^{-1}$. The XRF maps consist of a $31 \times 31$ raster using $20 \mu \mathrm{m}$ step size in both $X$ and $Y$ directions and the scale bar is the same for all the maps, indicating a normalized $\mathrm{Cu}-\mathrm{K} \alpha$ intensity between 0 and 0.13 .

concentration obtained via the electrochemical data is constant after the breaking point, which is indeed the case here. In contrast, the Co concentration calculated from the SR-XRF data increases for the three latter concentrations. An explanation could be that at these concentrations the modification procedure takes 9 voltammetric scans. It has been shown in previous work ${ }^{16}$ that at the beginning of the modification
(5 subsequent voltammetric scans in $6 \mathrm{mmol} \mathrm{L} \mathrm{L}^{-1}$ CoTSPc) there is a fast growth of electro-active species (monomers) on the surface in comparison with the electro-inactive species (dimers). This indicates that on the bare gold electrode, primarily monomers are adsorbed. According to this behaviour, it can be postulated that at the breaking point there is a concentration independent equilibrium between the constant 
Table 2 Overview of the SR-XRF data, including the number of the measurement, the data derived from the sum spectrum (resp. total Co or Cu intensity, the normalized intensity and the surface concentration) and the data calculated from the histograms (resp. mean of the histogram, the Co or $\mathrm{Cu}$ surface concentration, $\delta_{\text {tot, } \mathrm{r}}, \delta_{\text {stat, } \mathrm{r}}$ and $\delta_{\text {het, } \mathrm{r}}$ )

\begin{tabular}{|c|c|c|c|c|c|c|c|c|c|}
\hline & $\begin{array}{l}\text { Total Co/ } \\
\text { Cu intensity/ } \\
\text { counts s } \text { s }^{-1}\end{array}$ & $\begin{array}{l}\text { Normalized } \\
\text { intensity }\end{array}$ & $\begin{array}{l}\text { Surface } \\
\text { concentration/ } \\
\mu \mathrm{g} \mathrm{cm}^{-2}\end{array}$ & $\begin{array}{l}\delta_{\text {sum, }, \mathrm{r}} \\
(\%)\end{array}$ & $\begin{array}{l}\text { Mean } \\
\text { (histogram) }\end{array}$ & $\begin{array}{l}\text { Surface } \\
\text { concentration/ } \\
\mu \mathrm{g} \mathrm{cm}^{-2}\end{array}$ & $\begin{array}{l}\delta_{\text {tot, } \mathrm{r}} \\
(\%)\end{array}$ & $\begin{array}{l}\delta_{\text {stat, }, \mathrm{r}} \\
(\%)\end{array}$ & $\begin{array}{l}\delta_{\text {het, }} \\
(\%)\end{array}$ \\
\hline \multicolumn{10}{|c|}{ CoTSPc } \\
\hline 1 & 663 & 0.0744 & 0.333 & 0.37 & 0.068 & 0.31 & 11.8 & 1.3 & 11.8 \\
\hline 2 & 772 & 0.0923 & 0.423 & 0.36 & 0.085 & 0.38 & 6.5 & 1.2 & 6.4 \\
\hline 3 & 727 & 0.0907 & 0.395 & 0.37 & 0.085 & 0.38 & 7.8 & 1.2 & 7.7 \\
\hline 4 & 678 & 0.0933 & 0.393 & 0.35 & 0.083 & 0.37 & 10.8 & 1.3 & 10.7 \\
\hline 5 & 658 & 0.0735 & 0.342 & 0.37 & 0.068 & 0.31 & 14.0 & 1.3 & 14.0 \\
\hline 6 & 499 & 0.0594 & 0.279 & 0.37 & 0.055 & 0.26 & 7.5 & 1.5 & 7.3 \\
\hline 7 & 1602 & 0.1204 & 0.558 & 2.48 & 0.112 & 0.52 & 9.9 & 0.8 & 9.9 \\
\hline 8 & 1177 & 0.0904 & 0.429 & 3.24 & 0.096 & 0.46 & 16.7 & 0.9 & 16.7 \\
\hline 9 & 2316 & 0.1343 & 0.618 & 2.24 & 0.113 & 0.53 & 24.5 & 0.8 & 24.5 \\
\hline \multicolumn{10}{|c|}{ CuTSPc } \\
\hline 10 & 145 & 0.0366 & 0.092 & 12.4 & 0.031 & 0.09 & 10.2 & 2.8 & 9.8 \\
\hline 11 & 181 & 0.0427 & 0.108 & 12.4 & 0.036 & 0.10 & 10.4 & 2.5 & 10.0 \\
\hline 12 & 123 & 0.0355 & 0.103 & 12.4 & 0.029 & 0.08 & 9.0 & 3.2 & 8.4 \\
\hline 13 & 157 & 0.0427 & 0.119 & 12.4 & 0.034 & 0.09 & 19.0 & 2.8 & 18.8 \\
\hline 14 & 174 & 0.0585 & 0.143 & 12.4 & 0.048 & 0.13 & 22.7 & 2.6 & 22.6 \\
\hline 15 & 120 & 0.0723 & 0.192 & 12.4 & 0.063 & 0.17 & 12.6 & 3.1 & 12.1 \\
\hline 16 & 117 & 0.0555 & 0.168 & 12.4 & 0.041 & 0.11 & 15.6 & 3.4 & 15.3 \\
\hline 17 & 90 & 0.0375 & 0.114 & 10.4 & 0.021 & 0.07 & 16.3 & 4.5 & 15.7 \\
\hline 18 & 126 & 0.0415 & 0.125 & 9.6 & 0.024 & 0.08 & 21.9 & 3.7 & 21.5 \\
\hline
\end{tabular}

amounts of monomers and dimers. The surface area is the limiting factor according to the amount of monomer adsorbed on the surface, and there is a maximum amount of dimers adsorbed on the monomer layer. The continuous potential cycling after the breaking point causes further aggregation between the electro-active species. This means that there is a stabilization of the layer and that it is better defined. On this well defined layer, however, there is an increasing amount of dimer adsorbed after the breaking point. It is also noticed that $\delta_{\text {het }}$ on the Co concentration after the breaking point increases with increasing concentration, indicating an increase in heterogeneity. This can be explained by electro-inactivity of the dimers, ${ }^{16}$ which means that they are much less affected by the potential cycling.

\section{Comparison of the $\mathrm{Cu}$ surface concentrations on gold electrodes derived from SR-XRF and electrochemical data}

A similar experiment was performed using CuTSPc. Electrochemical and SR-XRF analyses of modified gold electrodes during 60 subsequent voltammetric scans as a function of the CuTSPc concentration in solution (between 1 and $16 \mathrm{mmol}$ $\mathrm{L}^{-1}$ ) were performed. Fig. 8 shows the plot of the deposited copper surface concentrations calculated using the three methods described above as a function of the CuTSPc concentration during modification. It is seen that these surface concentrations determined are more or less independent of the $\mathrm{CuTSPc}$ concentration in the range studied. In contrast with CoTSPc, the same copper surface concentrations are calculated from the electrochemical as well as the SR-XRF data. One can also observe that the surface concentrations calculated from the mean of the histogram and the sum spectrum are different from each other for the CuTSPc concentrations in solutions of $4,12,14$ and $16 \mathrm{mmol} \mathrm{L}^{-1}$. For 4 and $12 \mathrm{mmol} \mathrm{L}{ }^{-1} \mathrm{CuTSPc}$ concentration in solution, this can be explained by the distortion of the histogram from the fitted Gaussian (see Fig. 4). These histograms, indeed, show a relatively large number of high concentration pixels which can be caused by surface artifacts.

For the 14 and $16 \mathrm{mmol} \mathrm{L}^{-1}$ CuTSPc concentrations in solution, the difference in surface concentration can be explained by the energy shift of the incoming beam over $390 \mathrm{eV}$. As a result of this energy shift, the escape peak from the $\mathrm{Si}$ detector of the Rayleigh and Compton scatter interferes with the detected $\mathrm{Cu} \mathrm{K} \alpha$ which has, in this case, a similar intensity. This has a much higher influence on the (statistically more uncertain) individual pixel intensities than on the sum spectrum, which causes the mean to be underestimated together with the corresponding $\mathrm{Cu}$ surface concentration.

In contrast with $\mathrm{CoTSPc}$, there is no breaking point observed electrochemically for CuTSPc, and the surface concentrations calculated from the electrochemical data closely match those derived from the SR-XRF data. These differences in electrochemical behaviour can be explained by the difference in structure of the immobilized layer caused by the four coordination of the CuTSPc in comparison with the six coordination of the CoTSPc. The four coordination of the CuTSPc causes the molecules to approach very easily without the formation of bridged systems (e.g., with oxygen or hydroxyl particles) as is the case with CoTSPc. This makes the formed dimers (containing a $\mathrm{Cu}-\mathrm{Cu}$ bond), which are much higher in concentration than for CoTSPc, electrochemically active. Thus, during the modification process the monomers as well as the dimers are electrochemically deposited and active on the gold electrode. As a result, the electrochemical deposition causes the formation of a compact layer consisting of dimers as well as monomers, which makes electron tunnelling throughout the stacked aggregates possible. This may also 

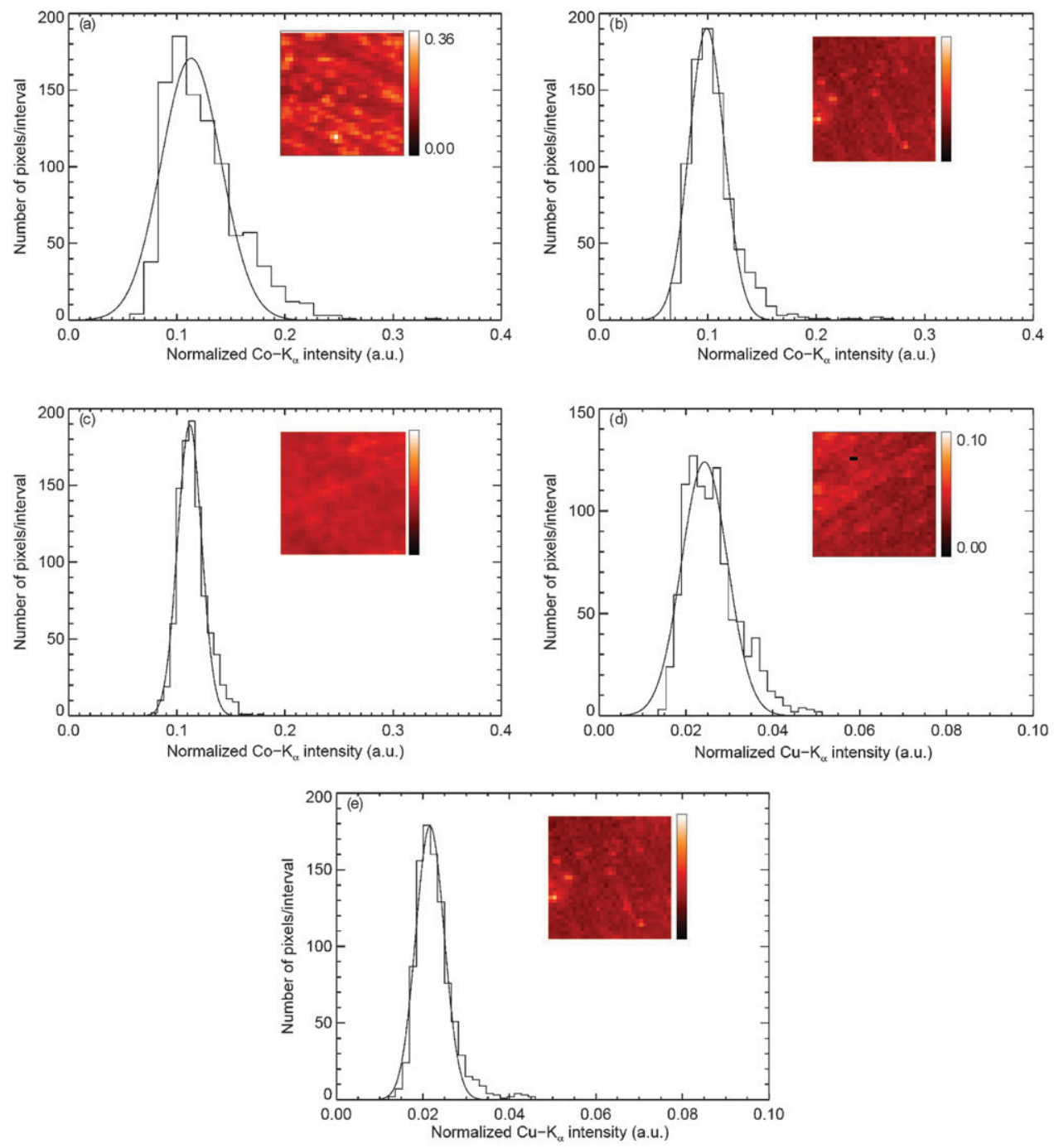

Fig. 5 Elemental maps of Co or Cu deposited with different CoTSPc (9 voltammetric scans) or CuTSPc (60 voltammetric scans) concentrations in solution and the corresponding histograms of the normalized $\mathrm{Co} \mathrm{K} \alpha$ or $\mathrm{Cu} \mathrm{K} \alpha$ intensities derived from this set of experiments: (a) $16 \mathrm{mmol}^{-1}$ CoTSPc, (b) $14 \mathrm{mmol} \mathrm{L}^{-1}$ CoTSPc, (c) $12 \mathrm{mmol} \mathrm{L}{ }^{-1}$ CoTSPc, (d) $16 \mathrm{mmol} \mathrm{L}^{-1} \mathrm{CuTSPc}$, (e) $14 \mathrm{mmol} \mathrm{L}^{-1}$ CuTSPc. The XRF maps consist of a $31 \times 31$ raster using $20 \mu \mathrm{m}$ step size in both $X$ and $Y$ directions. The scale bar is the same for all the Co maps (indicated in (a)) and another scale bar is valid for all the $\mathrm{Cu}$ maps (indicated in (d)).

explain the absence of a breaking point during the electrodeposition of the CuTSPc on the gold electrode since there is no interference from an electro-inactive species during the modification which makes the stacking of the phthalocyanine aggregates possible.

\section{Conclusions}

This paper describes the determination of the surface concentration and the (micro)-heterogeneity of thin layers of CoTSPc and $\mathrm{CuTSPc}$ on gold electrodes by means of synchrotron radiation X-ray micro-fluorescence on 20 and $600 \mu \mathrm{m}$ scales. CoTSPc or CuTSPc were deposited electrochemically on a gold electrode by recording successive cyclic voltammetric scans. The properties of the CoTSPc or CuTSPc layers were studied after modification using different phthalocyanine concentrations during the modification procedure.

It can be postulated that for the modification of a gold electrode with CoTSPc, there is an equilibrium between the constant amounts of electro-active species (monomer) and electro-inactive species (dimers) at the breaking point. The surface area is the limiting factor according to the amount of monomer adsorbed on the surface and there is a maximum constant amount of dimers adsorbed on the monomer layer. After the breaking point, the monomer layer stabilizes, increasing the free spaces for the dimers to adsorb.

For the modification of a gold electrode with CuTSPc it can be postulated that the monomers, as well as the dimers, are electrochemically active because of the absence of bridged dimers. This changes the modification in such a way that during the modification procedure stacked phthalocyanine 


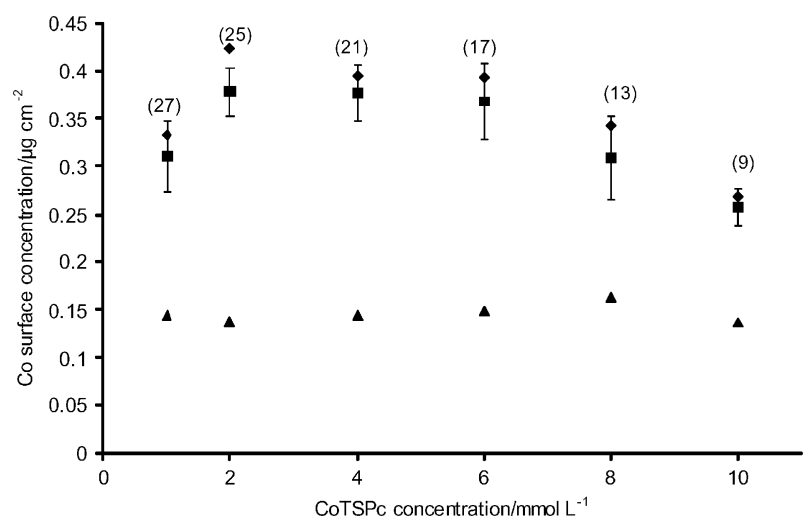

Fig. 6 Relationship between the Co surface concentrations calculated using the electrochemical data $(\boldsymbol{\Delta})$, the sum spectra $(\bullet)$ and using the mean of the histogram ( $\boldsymbol{\square})\left(\delta_{\text {tot }}\right.$ is shown as error bar) as a function of the CoTSPc concentration during the modification at the breaking point. The value in parentheses above each data point indicates the number of successive voltammetric scans.

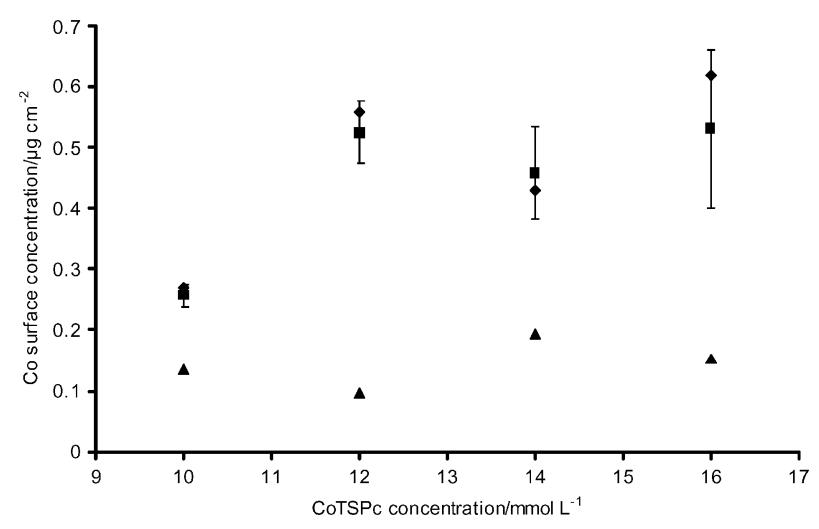

Fig. 7 Relationship between the Co surface concentrations calculated using the electrochemical data $(\boldsymbol{\Delta})$, the sum spectra $(\bullet)$ and the mean of the histogram ( $)$ ( $\delta_{\text {het }}$ is shown as error bar) as a function of the CoTSPc concentration after 9 successive voltammetric scans.

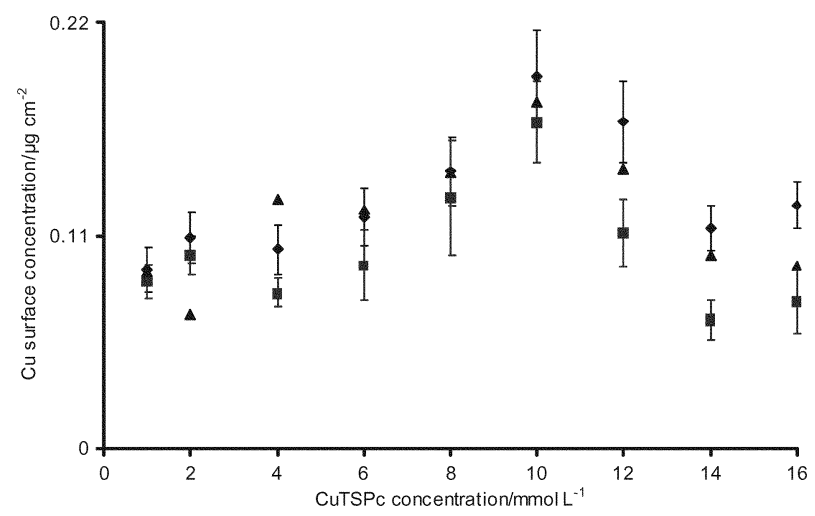

Fig. 8 Relationship between the $\mathrm{Cu}$ surface concentrations calculated using the electrochemical data $(\mathbf{\Lambda})$, the sum spectra $\left(\delta_{\text {sum }}\right.$ is shown as an error bar) $(\bullet)$ and using the mean of the histogram $(\boldsymbol{\square})\left(\delta_{\text {tot }}\right.$ is shown as an error bar) as a function of the CuTSPc concentration during the modification after 60 subsequent voltammetric scans. structure can be formed immediately and there is no stabilization of the layer needed.

\section{Acknowledgements}

This work was supported by the European CommunityResearch Infrastructure Action under the FP6 "Structuring the European Research Area" Programme (through the Integrated Infrastructure Initiative "Integrating Activity on Synchrotron and Free Electron Laser Science"). Ghent University (BOF) financed this work. In addition D. Bogaert, $\mathrm{H}$. Buschop, K. Leyssens, T. Schoonjans and B. Schotte (UGent) are acknowledged for their help during the SR-XRF measurements.

\section{References}

1 A. B. P. Lever, E. R. Milaeva and G. Speier in Phthalocyanines Properties and Applications, eds. C. C. Leznoff and A. B. P. Lever, VCH Publishers, Weinheim, 1993, vol. 3, ch. 1, pp. 5-69.

2 A. B. P. Lever, CHEMTECH, 1987, 17, 506-510.

3 M. Kato, Y. Nishioka, K. Kaifu, K. Kawamura and S. Ohno, Appl. Chem. Lett., 1985, 86, 196-197.

4 J. E. Kuder, J. Imaging Sci., 1988, 32, 51-56.

5 T. A. Temofonte and K. F. Schoch, J. Appl. Chem., 1989, 65, $1350-1355$.

6 K. J. Balkus, M. Eissa and R. Levado, J. Am. Chem. Soc., 1995, 117, 10753-10754.

7 A. Andreev, V. Ivanova, L. Prahov and I. D. Schopov, J. Mol. Catal., 1995, 95, 197-201.

8 K. De Wael, P. Westbroek and E. Temmerman, Electroanalysis, 2005, 17(3), 263-268.

9 N. Sehlotho, T. Nyokong, J. H. Zagal and F. Bedioui, Electrochim. Acta, 2006, 51, 5125-5130.

10 B. Agboola, K. I. Ozoemena and T. Nyokong, J. Mol. Catal., 2006, 248, 84-92.

11 Y.-C. Yang, J. R. Ward and R. P. Seiders, Inorg. Chem., 1985, 24, $1765-1769$.

12 M. Thamae and T. Nyokong, Polyhedron, 2002, 21, 133-140.

13 P. J. Camp, A. C. Jones, R. K. Neely and N. M. Speirs, J. Phys. Chem. A, 2002, 106, 10725-10732.

14 K. De Wael, P. Westbroek and E. Temmerman, J. Electroanal. Chem., 2004, 567, 167-173.

15 K. Peeters, K. De Wael, L. Vincze and A. Adriaens, Anal. Chem., 2005, 77, 5512-5519.

16 L. Kempenaers, C. De Koster, W. Van Borm and K. Janssens, Fresenius' J. Anal. Chem., 2001, 369, 733-737.

17 L. Kempenaers, K. Janssens, L. Vincze, B. Vekemans, A. Somogyi, M. Drakopoulos, A. Simionovici and F. Adams, Anal. Chem., 2002, 74, 5017-5026.

18 L. Kempenaers, L. Vincze and K. Janssens, Spectrochim. Acta, Part B, 2000, 55, 651-669.

19 K. Peeters, K. De Wael, A. Adriaens, G. Falkenberg and L. Vincze, Electrochem. Commun., 2005, 7, 1157-1162.

20 K. De Wael, K. Peeters, D. Bogaert, H. Buschop and A. Adriaens, J. Electroanal. Chem., in press.

21 G. Falkenberg, O. Clauss, A. Swiderski and T. Tschentscher, $X$-Ray Spectrom., 2001, 30, 170-173.

22 B. Vekemans, K. Janssens, L. Vincze, F. Adams and P. Vanespen, X-Ray Spectrom., 1994, 23, 278-285.

23 J. De Vries and B. Vrebos in Handbook of X-ray Spectrometry, eds. E. Van Grieken and A. Markowicz, Marcel Dekker, New York, 2nd edn., 2001, ch. 5, pp. 341-406.

24 W. H. McMaster, N. K. Delgrande, J. H. Mallet and J. H. Hubbel, Lawrence Radiation Laboratory report UCLR-50174, Section II, ref. 1, University of California, Berkeley, CA, 1968. 